\title{
TITLE:
}

\section{Quantum Hall liquid on a noncommutative superplane}

\author{
$\operatorname{AUTHOR}(S)$ :
}

Hasebe, $\mathrm{K}$

\section{CITATION:}

Hasebe, K. Quantum Hall liquid on a noncommutative superplane. PHYSICAL REVIEW D 2005, 72(10): 105017.

\section{ISSUE DATE:}

2005-11

URL:

http://hdl.handle.net/2433/50482

RIGHT:

Copyright 2005 American Physical Society 
PHYSICAL REVIEW D 72, 105017 (2005)

\title{
Quantum Hall liquid on a noncommutative superplane
}

\author{
Kazuki Hasebe* \\ Yukawa Institute for Theoretical Physics, Kyoto University, Kyoto 606-8502, Japan
}

(Received 30 March 2005; published 30 November 2005)

\begin{abstract}
Supersymmetric quantum Hall liquids are constructed on a noncommutative superplane. We explore a supersymmetric formalism of the Landau problem. In the lowest Landau level, there appear spinless bosonic states and spin-1/2 down fermionic states, which exhibit a superchiral property. It is shown that the Laughlin wave function and topological excitations have their superpartners. Similarities between supersymmetric quantum Hall systems and bilayer quantum Hall systems are discussed.
\end{abstract}

DOI: 10.1103/PhysRevD.72.105017

PACS numbers: 11.10.Nx, 11.30.Pb, 73.43.-f

\section{INTRODUCTION}

Over the past few years, accompanied with the developments of the noncommutative (NC) geometry and string theory, quantum Hall $(\mathrm{QH})$ systems have attracted increasing attention from particle physicists. (See [1,2], for instance.) It is well known that the underlying mathematical structure of QH systems is NC geometry, and QH systems manifest its exotic properties [3-6]. Based on the second Hopf map, a four-dimensional generalization of QH liquid was constructed in Ref. [7]. The system has higher dimensional analogues of the exotic structures of the twodimensional QH system, such as NC geometry, fractionally charged excitations, massless edge states, etc. Since then, many efforts have been devoted to the understanding of the four-dimensional $\mathrm{QH}$ liquid [8-16] and the construction of even higher dimensional QH systems [17-21]. The studies of higher dimensional QH systems have brought many fruitful developments in both particle physics and condensed matter physics. Particularly, spherical bound states of $\mathrm{D}$ branes in string theory were well investigated based on the setup of the fuzzy spheres in higher dimensional QH systems [22]. Three-dimensional reduction of four-dimensional $\mathrm{QH}$ effects gave a hint to the discovery of the spin-Hall effect [23], which has become one of the most rapidly growing topics in condensed matter physics.

Recently, it was discovered that the nonanticommutative (NAC) field theory is naturally realized on D branes in a Ramond-Ramond field or graviphoton background [2427]. Also, it has been shown that, in the supermatrix model, fluctuations on a fuzzy supersphere yield supersymmetric $\mathrm{NC}$ field theories [27]. Besides, some interesting relations between NAC geometry, Landau problems, and QH systems are reported [28-31]. Specifically, on a fuzzy supersphere, a supersymmetric extension of $\mathrm{QH}$ liquid was explicitly constructed in Ref. [32]. While mathematical properties of NAC theories have been well investigated [33-35], their emergent physical consequences have not

*Email address: hasebe@yukawa.kyoto-u.ac.jp been satisfactorily understood yet. The supersymmetric QH system provides a rare "physical" setup whose underlying mathematics is given by NAC geometry. Since twodimensional and higher dimensional $\mathrm{QH}$ systems manifest peculiar properties of $\mathrm{NC}$ geometry, it would be reasonable to expect that explorations of supersymmetric QH liquids may reveal yet unknown physical aspects of the NAC geometry. In this paper, by taking a planar limit of the fuzzy supersphere, we construct QH liquids on a NC superplane, and investigate physical properties in a NAC world.

This paper is organized as follows. In Sec. II, we review a systematic construction of a NC superplane from the fuzzy supersphere. It is shown that the NC superplane is realized by introducing the super gauge fields. In Sec. III, we develop Lagrangian and Hamiltonian formalisms for a one-particle system on the NC superplane. The system possesses (complex) $\mathcal{N}=2$ supersymmetry, one of which is dynamical and the other nondynamical. Another approach for a one-particle system on a NC plane with supersymmetry is found in Refs. [36,37], where a higher derivative term is introduced to be invariant under the Galilean boosts transformation. In Sec. IV, we analyze a supersymmetric Landau problem. In each of the higher Landau levels (LLs), there exists $\mathcal{N}=2$ supersymmetry, while in the lowest Landau level (LLL), only the $\mathcal{N}=1$ nondynamical supersymmetry remains valid. We explicitly construct radially symmetric orbit states, which form a "complete" basis in the LLL. These states are superholomorphic except for their exponential term, and show a superchiral property where not only the orbital rotation but also the spin polarization is chiral. In Sec. V, a Laughlin wave function and its superpartner on the NC superplane are derived. In Sec. VI, we present bosonic and fermionic topological excitations, and investigate their basic properties. In Sec. VII, we discuss a possible mapping from supersymmetric $\mathrm{QH}$ systems to bilayer $\mathrm{QH}$ systems. Section VIII is devoted to a summary and discussions. In Appendix A, magnetic translations on the superplane and their accompanied Aharonov-Bohm phases are summarized. In Appendix B, a supersymmetric extension of the $W_{\infty}$ algebra in the LLL is discussed. 


\section{NONCOMMUTATIVE SUPERPLANE}

Based on Ref. [28], we review an algebra on a NC superplane from the $O S p(1 \mid 2)$ algebra. The $O S p(1 \mid 2)$ algebra consists of five generators $L_{a}(a=1,2,3)$ and $L_{\alpha}(\alpha=1,2)$,

$$
\begin{aligned}
{\left[L_{a}, L_{b}\right] } & =i \epsilon_{a b c} L_{c}, \\
{\left[L_{a}, L_{\alpha}\right] } & =\frac{1}{2}\left(\sigma_{a}\right)_{\beta \alpha} L_{\beta}, \\
\left\{L_{\alpha}, L_{\beta}\right\} & =\frac{1}{2}\left(C \sigma_{a}\right)_{\alpha \beta} L_{a},
\end{aligned}
$$

where $\left\{\sigma_{a}\right\}$ are Pauli matrices and $C$ denotes a charge conjugation matrix $C=i \sigma_{2}$. With a given noncommutative scale $\alpha$, the coordinates on the fuzzy supersphere $S_{F}^{2 \mid 2}$ are identified with the $O S \mathrm{Sp}(1 \mid 2)$ generators by $X_{a}=\alpha L_{a}$ and $\Theta_{\alpha}=\alpha L_{\alpha}[31]$.

We apply a symmetric scaling to the $O S p(1 \mid 2)$ generators as

$$
\begin{aligned}
\left(L_{i}, L_{\alpha}\right) & \rightarrow\left(T_{i}, T_{\alpha}\right)=\epsilon\left(L_{i}, L_{\alpha}\right), \\
L_{3} & \rightarrow L_{\perp},
\end{aligned}
$$

where $i=1,2$. By taking the limit $\epsilon \rightarrow 0$, the $O S p(1 \mid 2)$ algebra reduces to the translation and rotation algebras on the superplane

$$
\begin{aligned}
{\left[T_{i}, T_{j}\right]=0,\left[T_{i}, L_{\perp}\right] } & =-i \epsilon_{i j} T_{j}, \\
{\left[T_{i}, T_{\alpha}\right] } & =0, \\
\left\{T_{\alpha}, T_{\beta}\right\}=0,\left[T_{\alpha}, L_{\perp}\right] & = \pm \frac{1}{2} T_{\alpha},
\end{aligned}
$$

where, in Eq. (2.3c), + corresponds to $\alpha=\theta_{1}$, and corresponds to $\alpha=\theta_{2}$. Equation (2.3a) represents the algebra of the two-dimensional Euclidean group. Similarly, Eq. (2.3c) may be regarded as the algebra of the symmetry group on the two-dimensional fermionic plane. The differential representation for the algebras (2.3) is given by

$$
\begin{aligned}
T_{i} & =-i \partial_{i}, \quad T_{\alpha}=-i \partial_{\alpha}, \\
L_{\perp} & =\left(\sigma_{2}\right)_{i j} x_{i} \partial_{j}+\frac{1}{2}\left(\sigma_{3}\right)_{\alpha \beta} \theta_{\alpha} \partial_{\beta} .
\end{aligned}
$$

Around the north pole on the fuzzy supersphere, $X_{3} \sim$ $\alpha j$ (where $j$ is a superspin index which specifies irreducible representations of the $O S p(1 \mid 2)$ group), the NC algebras on the fuzzy supersphere reduce to those on the NC superplane $R_{\mathrm{NC}}^{2 \mid 2}$,

$$
\begin{aligned}
{\left[\hat{X}_{1}, \hat{X}_{2}\right] } & =-i, \\
{\left[\hat{X}_{i}, \hat{\Theta}_{\alpha}\right] } & =0, \\
\left\{\hat{\Theta}_{1}, \hat{\Theta}_{2}\right\} & =1,
\end{aligned}
$$

where we have defined the dimensionless coordinates as $\hat{X}_{i}=\frac{1}{\alpha \sqrt{j}} X_{i}, \hat{\Theta}_{\alpha}=\frac{\sqrt{2}}{\alpha \sqrt{j}} \Theta_{\alpha}$. (More general contractions, including asymmetric scaling, are found in Ref. [28].)
The bosonic coordinates and the fermionic coordinates are completely decoupled unlike the fuzzy supersphere case. The algebra (2.5a) is equivalent to that on the $\mathrm{NC}$ bosonic plane. The original QH systems on the $\mathrm{NC}$ bosonic plane have already been well investigated as found in Ref. [38]. In the following, we include the known results on the NC bosonic plane for a complete description.

A physical setup for the NC superplane is realized by introducing super gauge fields. We consider a constant magnetic strength made by a bosonic gauge field and a fermionic gauge field as

$$
\begin{aligned}
& B=-i\left(\sigma_{2}\right)_{i j} \partial_{i} A_{j}=-\epsilon_{i j} \partial_{i} A_{j}, \\
& B=-i\left(\sigma_{3}\right)_{\alpha \beta} \partial_{\alpha}\left(C_{\beta \gamma} A_{\gamma}\right)=-i\left(\sigma_{1}\right)_{\alpha \beta} \partial_{\alpha} A_{\beta} .
\end{aligned}
$$

It is apparent that there exists a $U(1)$ gauge degree of freedom, $A_{i} \rightarrow A_{i}+\partial_{i} \xi$ and $A_{\alpha} \rightarrow A_{\alpha}+\partial_{\alpha} \xi$. The covariant momenta are given by

$$
\begin{aligned}
P_{i} & =-i\left(\partial_{i}+i A_{i}\right), \\
P_{\alpha} & =i\left(\partial_{\alpha}+i A_{\alpha}\right) .
\end{aligned}
$$

With these covariant momenta, the center-of-mass coordinates are defined as

$$
\begin{gathered}
X_{i}=x_{i}+i \ell_{B}^{2}\left(\sigma_{2}\right)_{i j} P_{j}, \\
\Theta_{\alpha}=\theta_{\alpha}-i \ell_{B}^{2}\left(\sigma_{1}\right)_{\alpha \beta} P_{\beta},
\end{gathered}
$$

where $\ell_{B} \equiv 1 / \sqrt{B}$ is the magnetic length.

The center-of-mass coordinates and the covariant momenta are completely decoupled, and satisfy the super Heisenberg-Weyl algebra individually,

$$
\begin{aligned}
{\left[P_{i}, P_{j}\right] } & =-\frac{1}{\ell_{B}^{2}}\left(\sigma_{2}\right)_{i j}, \\
{\left[P_{i}, P_{\alpha}\right] } & =0, \\
\left\{P_{\alpha}, P_{\beta}\right\} & =\frac{1}{\ell_{B}^{2}}\left(\sigma_{1}\right)_{\alpha \beta},
\end{aligned}
$$

and

$$
\begin{aligned}
{\left[X_{i}, X_{j}\right] } & =\ell_{B}^{2}\left(\sigma_{2}\right)_{i j}, \\
{\left[X_{i}, \Theta_{\alpha}\right] } & =0, \\
\left\{\Theta_{\alpha}, \Theta_{\beta}\right\} & =\ell_{B}^{2}\left(\sigma_{1}\right)_{\alpha \beta} .
\end{aligned}
$$

The set of algebras (2.10) is consistent with Eq. (2.5). In the LLL limit $(B \rightarrow \infty)$, it is easily seen from Eq. (2.8) that the particle position $\left(x_{i}, \theta_{\alpha}\right)$ reduces to the center-of-mass coordinate operator $\left(X_{i}, \Theta_{\alpha}\right)$, and the superplane under the strong supermagnetic field is identified with the NC superplane.

The angular momentum (2.4b) can be rewritten in terms of the covariant momenta and the center-of-mass coordinates as 
QUANTUM HALL LIQUID ON A NONCOMMUTATIVE ...

$$
\begin{aligned}
L_{\perp}= & \frac{1}{2 \ell_{B}^{2}}\left(X_{i}^{2}+\frac{1}{2} C_{\alpha \beta} \Theta_{\alpha} \Theta_{\beta}\right) \\
& -\frac{1}{2} \ell_{B}^{2}\left(P_{i}^{2}+\frac{1}{2} C_{\alpha \beta} P_{\alpha} P_{\beta}\right) .
\end{aligned}
$$

The center-of-mass coordinates $\left(X_{i}, \Theta_{\alpha}\right)$ and the covariant momenta $\left(P_{i}, P_{\alpha}\right)$ form a closed algebra with $L_{\perp}$, individually,

$\left[L_{\perp}, X_{i}\right]=-\left(\sigma_{2}\right)_{i j} X_{j}, \quad\left[L_{\perp}, \Theta_{\alpha}\right]=\frac{1}{2}\left(\sigma_{3}\right)_{\alpha \beta} \Theta_{\beta}$,

$\left[L_{\perp}, P_{i}\right]=\left(\sigma_{2}\right)_{i j} P_{j}, \quad\left[L_{\perp}, P_{\alpha}\right]=-\frac{1}{2}\left(\sigma_{3}\right)_{\alpha \beta} P_{\beta}$.

Because of the existence of two sets of the super Heisenberg-Weyl algebras, two sets of supersymmetric harmonic oscillators are naturally defined. The bosonic creation and annihilation operators are given by

$a \equiv \frac{\ell_{B}}{\sqrt{2}}\left(P_{x}+i P_{y}\right), \quad a^{\dagger} \equiv \frac{\ell_{B}}{\sqrt{2}}\left(P_{x}-i P_{y}\right)$,

$b \equiv \frac{1}{\sqrt{2} \ell_{B}}(X-i Y), \quad b^{\dagger} \equiv \frac{1}{\sqrt{2} \ell_{B}}(X+i Y)$,

which satisfy $\left[a, a^{\dagger}\right]=\left[b, b^{\dagger}\right]=1$. Other commutators become zeros. Similarly, the fermionic creation and annihilation operators are given by

$$
\begin{array}{rlrl}
\alpha \equiv \ell_{B} P_{\theta_{2}}, & & \alpha^{\dagger} \equiv \ell_{B} P_{\theta_{1}}, \\
\beta \equiv \frac{1}{\ell_{B}} \Theta_{2}, & \beta^{\dagger} \equiv \frac{1}{\ell_{B}} \Theta_{1},
\end{array}
$$

which satisfy $\left\{\alpha, \alpha^{\dagger}\right\}=\left\{\beta, \beta^{\dagger}\right\}=1$. Other anticommutators are zeros. With use of supersymmetric harmonic oscillators, the angular momentum can be written as

$$
L_{\perp}=\left(b^{\dagger} b+\frac{1}{2} \beta^{\dagger} \beta\right)-\left(a^{\dagger} a+\frac{1}{2} \alpha^{\dagger} \alpha\right) .
$$

Thus, the $b$ quantum acquires the angular momentum by 1 , while the $\beta$ quantum acquires the angular momentum by $1 /$ 2.

It is convenient to fix the gauge freedom as the symmetric gauge,

$$
A_{i}=i\left(\sigma_{2}\right)_{i j} x_{j} \frac{B}{2}, \quad A_{\alpha}=i\left(\sigma_{1}\right)_{\alpha \beta} \theta_{\beta} \frac{B}{2} .
$$

These expressions are obtained by expanding the supermonopole gauge fields [31] around the north pole on the supersphere. The field strengths become

$$
\begin{aligned}
F_{i j} & =\partial_{i} A_{j}-\partial_{j} A_{i}=-i B\left(\sigma_{2}\right)_{i j}, \\
F_{i \alpha} & =\partial_{i} A_{\alpha}-\partial_{\alpha} A_{i}=0, \\
F_{\alpha \beta} & =\partial_{\alpha} A_{\beta}+\partial_{\beta} A_{\alpha}=i B\left(\sigma_{1}\right)_{\alpha \beta} .
\end{aligned}
$$

In the symmetric gauge, the creation and annihilation operators (2.13) and (2.14) read as
PHYSICAL REVIEW D 72, 105017 (2005)

$$
\begin{array}{ll}
a=-\frac{i}{\sqrt{2}}\left(z+\partial^{*}\right), & a^{\dagger}=\frac{i}{\sqrt{2}}\left(z^{*}-\partial\right), \\
b=\frac{1}{\sqrt{2}}\left(z^{*}+\partial\right), & b^{\dagger}=\frac{1}{\sqrt{2}}\left(z-\partial^{*}\right),
\end{array}
$$

and

$\alpha=-\frac{i}{\sqrt{2}}\left(\theta-\partial_{\theta}^{*}\right), \quad \alpha^{\dagger}=-\frac{i}{\sqrt{2}}\left(\theta^{*}-\partial_{\theta}\right)$,

$\beta=\frac{1}{\sqrt{2}}\left(\theta^{*}+\partial_{\theta}\right), \quad \beta^{\dagger}=\frac{1}{\sqrt{2}}\left(\theta+\partial_{\theta}^{*}\right)$,

where we have used dimensionless complex coordinates and derivatives,

$$
\begin{aligned}
z & =\frac{1}{2 \ell_{B}}(x+i y), & z^{*} & =\frac{1}{2 \ell_{B}}(x-i y), \\
\partial & =\ell_{B}\left(\partial_{x}-i \partial_{y}\right), & \partial^{*} & =\ell_{B}\left(\partial_{x}+i \partial_{y}\right),
\end{aligned}
$$

and

$$
\begin{aligned}
\theta & =\frac{1}{\sqrt{2} \ell_{B}} \theta_{1}, & \theta^{*} & =\frac{1}{\sqrt{2} \ell_{B}} \theta_{2}, \\
\partial_{\theta} & =\sqrt{2} \ell_{B} \partial_{\theta_{1}}, & \partial_{\theta}^{*} & =\sqrt{2} \ell_{B} \partial_{\theta_{2}} .
\end{aligned}
$$

\section{ONE-PARTICLE HAMILTONIAN AND SUPERSYMMETRY}

We develop a Lagrangian formalism for one particle in the presence of super gauge fields. The Lagrangian may be given by

$$
L=\frac{M}{2}\left(\dot{x}_{i}^{2}+C_{\alpha \beta} \dot{\theta}_{\alpha} \dot{\theta}_{\beta}\right)-\left(A_{i} \dot{x}_{i}+A_{\alpha} \dot{\theta}_{\alpha}\right) .
$$

In the LLL limit, the kinetic term is quenched, and the Lagrangian (3.1) reduces to

$$
L_{\mathrm{eff}}=-A_{i} \dot{x}_{i}-A_{\alpha} \dot{\theta}_{\alpha} .
$$

The canonical momenta are derived as

$$
\begin{aligned}
& p_{i}=\frac{\partial}{\partial \dot{x}_{i}} L_{\mathrm{eff}}=-A_{i}=-i\left(\sigma_{2}\right)_{i j} x_{j} \frac{B}{2}, \\
& p_{\alpha}=\frac{\partial}{\partial \dot{\theta}_{\alpha}} L_{\mathrm{eff}}=A_{\alpha}=i\left(\sigma_{1}\right)_{\alpha \beta} \theta_{\beta} \frac{B}{2},
\end{aligned}
$$

where the symmetric gauge was used in the last equations. By imposing the commutation relations to canonical variables

$$
\begin{gathered}
{\left[x_{i}, p_{j}\right]=i \delta_{i j},} \\
\left\{\theta_{\alpha}, p_{\beta}\right\}=i \delta_{\alpha \beta},
\end{gathered}
$$

we obtain the $\mathrm{NC}$ relations

$$
\begin{gathered}
{\left[x_{i}, x_{j}\right]=\ell_{B}^{2}\left(\sigma_{2}\right)_{i j},} \\
\left\{\theta_{\alpha}, \theta_{\beta}\right\}=\ell_{B}^{2}\left(\sigma_{1}\right)_{\alpha \beta} .
\end{gathered}
$$


These relations are what we have already obtained in Eq. (2.10). Then, it would be reasonable to adopt Eq. (3.1) as the Lagrangian for the present system.

The equations of motions are derived as

$$
\begin{aligned}
M \ddot{x}_{i} & =\epsilon_{i j} B \dot{x}_{j}, \\
M \ddot{\theta}_{\alpha} & =-i\left(\sigma_{3}\right)_{\alpha \beta} B \dot{\theta}_{\beta},
\end{aligned}
$$

which represent cyclotron motions for bosonic and fermionic degrees of freedom. As we shall discuss in the next section, the fermionic variables $\left\{\theta_{\alpha}\right\}$ are related to the spin degrees of freedom. With the definition of the spin $S_{a}=$ $-i \frac{M}{2} \theta_{\alpha}\left(\sigma_{a} C\right)_{\alpha \beta} \dot{\theta}_{\beta}$, Eq. (3.6b) implies the spin precession motion,

$$
\dot{S}_{i}=-\epsilon_{i j} S_{j} B .
$$

The Lagrangian (3.1) apparently possesses translational symmetries on both the bosonic plane and the fermionic plane. The Noether charges accompanied by the translational symmetries are obtained as

$$
\begin{aligned}
\mathcal{P}_{i} & =M \dot{x}-B \epsilon_{i j} x_{j}, \\
\mathcal{P}_{\alpha} & =M C_{\alpha \beta} \dot{\theta}_{\beta}+i B\left(\sigma_{1}\right)_{\alpha \beta} \theta_{\beta},
\end{aligned}
$$

which are total momenta. The first terms on the right-hand sides in Eq. (3.8) represent the particle momenta, and the second terms represent the field momenta. The total momenta are related to the center-of-mass coordinates as

$$
\mathcal{P}_{i}=-B \epsilon_{i j} X_{j}, \mathcal{P}_{\alpha}=B\left(\sigma_{1}\right)_{\alpha \beta} \Theta_{\beta} .
$$

Hence, the center-of-mass coordinates are conserved quantities and essentially act as translational generators on the NC superplane.

Next, we develop a Hamiltonian formalism. The canonical momenta are given by

$$
\begin{aligned}
& p_{i}=\frac{\partial}{\partial \dot{x}_{i}} L=M \dot{x}_{i}-A_{i}, \\
& p_{\alpha}=\frac{\partial}{\partial \dot{\theta}_{\alpha}} L=M C_{\alpha \beta} \dot{\theta}_{\beta}+A_{\alpha},
\end{aligned}
$$

and the Hamiltonian is constructed as

$$
H=\dot{x}_{i} p_{i}+\dot{\theta}_{\alpha} p_{\alpha}-L=\frac{1}{2 M}\left(P_{i}^{2}+C_{\alpha \beta} P_{\alpha} P_{\beta}\right),
$$

where we have used the covariant momenta (2.7).

With use of creation and annihilation operators, two sets of supercharges are naturally defined as

$$
\begin{aligned}
& Q \equiv a^{\dagger} \alpha, \quad Q^{\dagger} \equiv \alpha^{\dagger} a, \\
& \tilde{Q} \equiv b^{\dagger} \beta, \quad \tilde{Q}^{\dagger} \equiv \beta^{\dagger} b,
\end{aligned}
$$

and the Hamiltonian (3.11) is expressed as

$$
H=\omega\left(a^{\dagger} a+\alpha^{\dagger} \alpha\right)=\omega\left\{Q, Q^{\dagger}\right\} .
$$

Thus, the supercharges $\left(Q, Q^{\dagger}\right)$ generate a dynamical supersymmetry. This Hamiltonian commutes with four supercharges, and the system possesses (complex) $\mathcal{N}=$ 2 supersymmetry. Some comments are added here. The Hamiltonian (3.13) is identical to the one used in the onedimensional supersymmetric harmonic oscillator system [39]. However, the one-dimensional harmonic oscillator system possesses $\mathcal{N}=1$ supersymmetry only, while the present system possesses $\mathcal{N}=2$ supersymmetry. (See also Sec. VII.) The anticommutator of $\left(\tilde{Q}, \tilde{Q}^{\dagger}\right)$ gives the radius on the $\mathrm{NC}$ superplane as

$$
\begin{aligned}
2 \ell_{B}^{2}\left\{\tilde{Q}, \tilde{Q}^{\dagger}\right\} & =2 \ell_{B}^{2}\left(b^{\dagger} b+\beta^{\dagger} \beta\right) \\
& =X_{i}^{2}+C_{\alpha \beta} \Theta_{\alpha} \Theta_{\beta} \equiv R^{2} .
\end{aligned}
$$

This expression implies that the eigenvalue of the radius operator $R^{2}$ takes a semipositive value, and the supersymmetry generated by $\left(\tilde{Q}, \tilde{Q}^{\dagger}\right)$ is a nondynamical one. Since $R^{2}$ commutes with the four supercharges, the $\mathcal{N}=2$ supermultiplet has not only an identical energy but also an identical eigenvalue of the radius operator.

The Hamiltonian and the radius operator commute with the angular momentum. Then, the four components of the $\mathcal{N}=2$ supermultiplet can be taken as simultaneous eigenstates of the angular momentum. The angular momentum and the supercharges satisfy the commutation relations

$$
\begin{aligned}
& {\left[L_{\perp}, Q\right]=-\frac{1}{2} Q, \quad\left[L_{\perp}, Q^{\dagger}\right]=\frac{1}{2} Q^{\dagger},} \\
& {\left[L_{\perp}, \tilde{Q}\right]=\frac{1}{2} \tilde{Q}, \quad\left[L_{\perp}, \tilde{Q}^{\dagger}\right]=-\frac{1}{2} \tilde{Q}^{\dagger} .}
\end{aligned}
$$

Thus, the supersymmetric transformations change the eigenvalue of the angular momentum by $1 / 2$.

\section{SUPERSYMMETRIC LANDAU PROBLEM}

The energy spectrum of the Hamiltonian (3.13) reads as

$$
E_{n}=\omega n,
$$

where $n=0,1,2, \ldots$ indicates the LL in the supersymmetric Landau problem. The zero-point energy is canceled due to the existence of the supersymmetry. The higher LLs are doubly degenerate compared to the LLL. The eigenvalue of the radius operator (3.14) is given by

$$
R_{m}=\sqrt{2 m} \ell_{B},
$$

where $m=0,1,2, \ldots$ indicates the radially symmetric orbits. The four components for the $\mathcal{N}=2$ supermultiplet with energy (4.1) and radius (4.2) are constructed as

$$
\begin{aligned}
& \frac{1}{\sqrt{n ! m !}}\left(a^{\dagger}\right)^{n}\left(b^{\dagger}\right)^{m}|0\rangle, \\
& \frac{1}{\sqrt{n !(m-1) !}}\left(a^{\dagger}\right)^{n} \beta^{\dagger}\left(b^{\dagger}\right)^{m-1}|0\rangle, \\
& \frac{1}{\sqrt{(n-1) ! m !}} \alpha^{\dagger}\left(a^{\dagger}\right)^{n-1}\left(b^{\dagger}\right)^{m}|0\rangle, \\
& \frac{1}{\sqrt{(n-1) !(m-1) !}} \alpha^{\dagger}\left(a^{\dagger}\right)^{n-1} \beta^{\dagger}\left(b^{\dagger}\right)^{m-1}|0\rangle .
\end{aligned}
$$


At the same time, they are eigenstates of the angular momenta $L_{\perp}$ with different eigenvalues, $l=m-n, m-$ $n-\frac{1}{2}, m-n+\frac{1}{2}$, and $m-n$, respectively. Here, we give a physical interpretation of these states. Because they have the identical energy and the radius, they may represent four particle states, which are on the same radially symmetric orbit, and rotate around the origin with the same frequency. Hence, they should carry the same orbital angular momentum, while their eigenvalues of the angular momentum $L_{\perp}$ are different. This discrepancy is solved by noticing that $L_{\perp}$ represents the total angular momentum, and each of the four particle states carries the intrinsic spin as well as the orbital angular momentum. Namely, the components of the $\mathcal{N}=2$ supermultiplet (4.3) are interpreted as the four particle states which have the identical orbital angular momentum $m-n$, and, simultaneously, have different spins $0,-1 / 2,1 / 2$, and 0 , respectively. Thus, two of them (4.3a) and (4.3d) are interpreted as spinless bosons, and the other two (4.3b) and (4.3c) are interpreted as spin$1 / 2$ down and up fermions. As suggested by Eq. (3.15), the $\mathcal{N}=2$ supersymmetry changes their spins by $1 / 2$, and transforms the bosons to the fermions and vice versa [Fig. 1]. It is noted that, in general, supersymmetric quantum mechanical models do not deal with a real bosonfermion symmetry [40], while supersymmetric quantum Hall systems deal with a real boson-fermion symmetry.

Each Hilbert space of the higher LL possesses the $\mathcal{N}=$ 2 supersymmetry, because $n$th $(n \geq 1)$ LL is spanned by $\mathcal{N}=2$ supermultiplets (4.3) with fixed $n$, while, in the

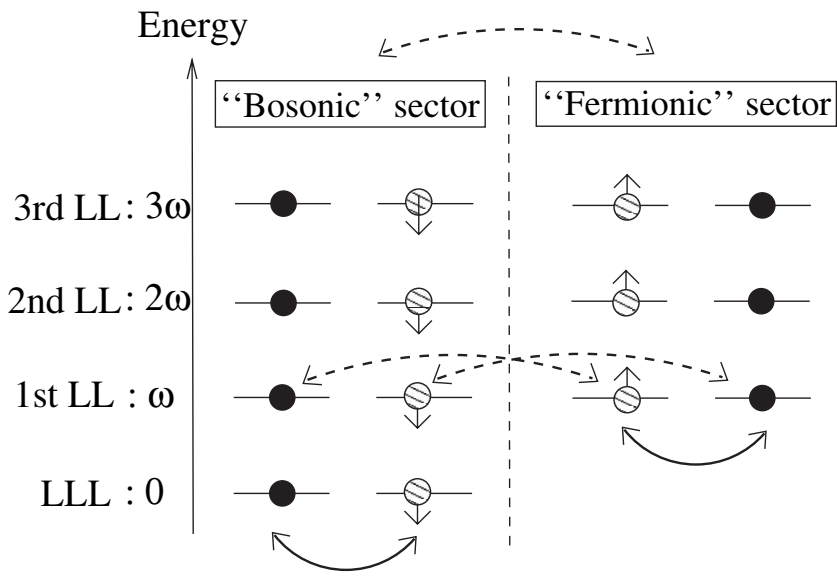

FIG. 1. The left sector about the vertical dashed axis is a "bosonic sector" for the dynamical supersymmetry, and the right sector is a "fermionic sector." The curved solid arrows represent the nondynamical supersymmetric transformation generated by $\left(\tilde{Q}, \tilde{Q}^{\dagger}\right)$, while the curved dashed arrows represent the dynamical supersymmetry transformation generated by $\left(Q, Q^{\dagger}\right)$. In each of the higher LLs, there are spinless, spin- $1 / 2$ up, and spin-1/2 down particles due to the existence of the $\mathcal{N}=2$ supersymmetry, while, in the LLL, the system possesses only $\mathcal{N}=1$ nondynamical supersymmetry, and there appear only spinless and spin-1/2 down particles.
LLL, only the nondynamical supersymmetry $\mathcal{N}=1$ remains valid, because the LLL is the "vacuum" for the $\mathcal{N}=1$ dynamical supersymmetry. In fact, in the LLL, the Hilbert space is spanned only by the $\mathcal{N}=1$ nondynamical superpartners

$$
\begin{aligned}
|m+1 / 2\rangle & =\frac{1}{\sqrt{m !}} \beta^{\dagger}\left(b^{\dagger}\right)^{m}|0\rangle, \\
|m+1\rangle & =\frac{1}{\sqrt{(m+1) !}}\left(b^{\dagger}\right)^{m+1}|0\rangle,
\end{aligned}
$$

(and the vacuum $|0\rangle$ ). In the symmetric gauge, with expression of the vacuum $\psi_{0}=\sqrt{(1 / \pi)} e^{-|z|^{2}-\theta \theta^{*}}$, they are represented as

$$
\begin{aligned}
\psi_{m+1 / 2} & =\sqrt{\frac{2^{m+1}}{\pi m !} z^{m} \theta e^{-|z|^{2}-\theta \theta^{*}},} \\
\psi_{m+1} & =\sqrt{\frac{2^{m+1}}{\pi(m+1) !} z^{m+1} e^{-|z|^{2}-\theta \theta^{*}} .}
\end{aligned}
$$

The "complete relation" in the LLL is obtained as

$$
\begin{aligned}
& \sum_{m \in 0, \mathbb{N} / 2} \psi_{m}\left(z, z^{*}, \theta, \theta^{*}\right) \psi_{m}^{*}\left(z^{\prime}, z^{\prime *}, \theta^{\prime}, \theta^{\prime *}\right) \\
& =\frac{1}{\pi} e^{-\left(|z|^{2}+\theta \theta^{*}\right)-\left(\left|z^{\prime}\right|^{2}+\theta^{\prime} \theta^{\prime *}\right)-2\left(z^{\prime *} z+\theta^{\prime *} \theta\right)} .
\end{aligned}
$$

These states are holomorphic about $z$ and $\theta$, i.e. superholomorphic except for their exponential term. They have angular momenta $m+1 / 2$ and $m+1$, respectively, and are localized on the same radially symmetric orbit with radius $R_{m+1}$. This reminds us of the situation where two particles, one of which has spin- 0 and the other spin- $1 / 2$ down, rotate on a plane with the same radius [Fig. 2]. There appear no spin-1/2 up fermions in the LLL, and the system shows the superchirality, where not only the orbital rotations but also the spin rotations are chiral. In the higher LLs, there are both spin-1/2 up and down fermions, and the system is nonchiral. (See Fig. 1.)

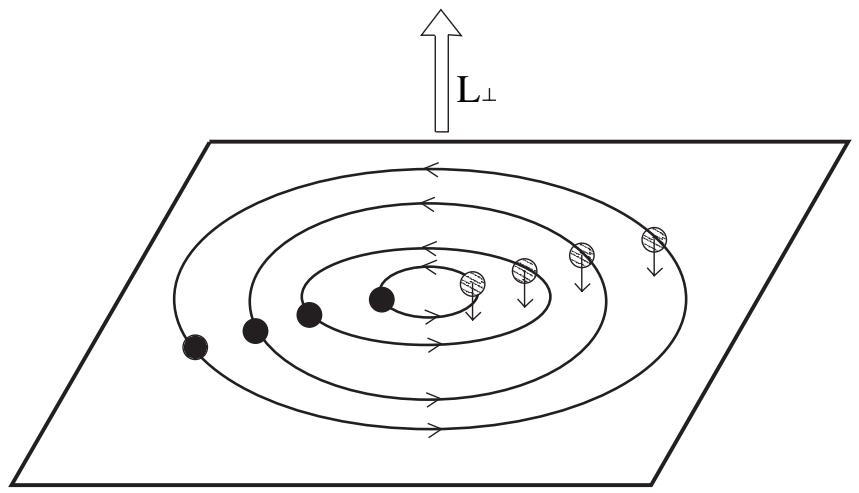

FIG. 2. There are spinless bosons and spin-1/2 down fermions in the LLL. They are on the radially symmetric orbits, and rotate around the origin with the same frequency. 


\section{LAUGHLIN WAVE FUNCTION AND ITS SUPERPARTNER}

We construct a Laughlin wave function in the supersymmetric framework, by demanding the following conditions as in the original case [41]. The Laughlin wavefunction (i) is an eigenstate of $L_{\perp}$, and (ii) possesses the translational symmetries on the superplane up to its exponential factor. We also postulate that the Laughlin wave function on the NC superplane is composed of a product of the bosonic part and the fermionic part. It may be natural to use the original Laughlin wave function as the bosonic part. With respect to the fermionic part, the Vandermonde determinant vanishes due to the nilpotency of the Grassmann number, $\prod_{i<j}^{N}\left(\theta_{i}-\theta_{j}\right)=0$ for $N \geq 3$, and $\left(\theta_{i}-\theta_{j}\right)^{m}=0$ for $m \geq 2$. Then, the Laughlin wave function on the NC superplane is simply given by

$$
\Psi_{\text {Llin }}=\prod_{p<q}^{N}\left(z_{p}-z_{q}\right)^{m} e^{-\sum_{p}\left(\left|z_{p}\right|^{2}+\left(\theta \theta^{*}\right)_{p}\right)},
$$

where $N$ denotes the number of particles. Apparently, $\Psi_{\text {Llin }}$ lives in the LLL, and is an eigenstate of $L_{\perp}$ with eigenvalue $m N(N-1) / 2$. Thus, $N$ particles described by $\Psi_{\text {Llin }}$ are spinless particles, which rotate on the radially symmetric orbits in order from the origin. Intriguingly, $\Psi_{\text {Llin }}$ has its superpartner $\Psi_{\text {sLlin }}$ unlike the Laughlin-Haldane wave function on the supersphere [32]. This stems from the decoupling between $X_{i}$ and $\Theta_{\alpha}(2.10 \mathrm{~b})$ on the NC superplane. The superpartner $\Psi_{\text {sLlin }}$ is related to $\Psi_{\text {Llin }}$ by the nondynamical supersymmetry, and is explicitly given by

$$
\Psi_{\text {sLlin }}=\sum_{p<q}\left(\frac{\theta_{p}-\theta_{q}}{z_{p}-z_{q}}\right) \cdot \Psi_{\text {Llin }}
$$

which has the angular momentum $(m N(N-1)-1) / 2$. It is noted that $\Psi_{\text {sLlin }}$ is not simply expressed as a product of a bosonic part and a fermionic part. The $N$-particle state described by $\Psi_{\text {sLlin }}$ is a superposition of all possible states where the $(N-1)$ spinless particles and one spin-1/2 down particle rotate on the radially symmetric orbits in order from the origin. With the definition of the filling factor $\nu \equiv$ $N /\left[A /\left(2 \pi \ell_{B}^{2}\right)\right]$ (where $A$ denotes the area on the superplane), $\Psi_{\text {Llin }}$ and $\Psi_{\text {sLlin }}$ may become two degenerate ground states of the supersymmetric QH systems at $\nu=$ $1 / m$, because they should have an identical energy due to the supersymmetry.

The density of $\Psi_{\text {Llin }}$ is

$$
\Psi_{\text {Llin }}^{*} \Psi_{\text {Llin }}=e^{-(2 / m) W}
$$

where $W$ is interpreted as the supersymmetric extension of the plasma potential,

$$
\begin{aligned}
W= & -\frac{m^{2}}{2} \sum_{p<q} \ln \left|(x+i y)_{p}-(x+i y)_{q}\right|^{2} \\
& -\frac{m B}{4} \sum_{p}\left(|x+i y|^{2}+2 \theta_{1} \theta_{2}\right)_{p} .
\end{aligned}
$$

The first term represents the interaction between particles with negative charge $m$ on the superplane. The second term is interpreted as a background made by unit positive charged particles which are uniformly distributed on the superplane with density $\rho_{\Phi}=1 / 2 \pi \ell_{B}^{2}$. This plasma analogy suggests that the state described by $\Psi_{\text {Llin }}$ becomes energetically favorable at $\nu=1 / m$, and fundamental excitations carry a fractional charge $1 / m$ as in the original case [41].

\section{HALL CURRENTS AND EXCITED STATES}

The Hall currents on the superplane are expressed as

$$
\begin{aligned}
I_{i} & =\frac{d}{d t} X_{i}=-i\left[X_{i}, V\right]=\epsilon_{i j} \ell_{B}^{2} E_{j}, \\
I_{\alpha} & =\frac{d}{d t} \Theta_{\alpha}=-i\left[\Theta_{\alpha}, V\right]=i \ell_{B}^{2}\left(\sigma_{3}\right)_{\alpha \beta} E_{\beta},
\end{aligned}
$$

where $\left\{E_{i}\right\}$ and $\left\{E_{\alpha}\right\}$ are bosonic and fermionic electric fields defined by $E_{i} \equiv-\partial_{i} V$ and $E_{\alpha} \equiv-C_{\alpha \beta} \partial_{\beta} V$. The Hall currents are orthogonal to the electric fields individually,

$$
E_{i} I_{i}=C_{\alpha \beta} E_{\alpha} I_{\beta}=0
$$

As suggested by the existence of the bosonic and fermionic Hall currents, there are two kinds of quasiholes, one of which is bosonic and the other fermionic. They are superpartners, and are constructed by operating the creation operators

$$
A_{B}^{\dagger}=\prod_{p} z_{p}, \quad A_{F}^{\dagger}=\prod_{p} \theta_{p},
$$

on the Laughlin wave function $\Psi_{\text {Llin }}$. The operators satisfy the commutation relations with the radius operator as

$$
\left[R^{2}, A_{B}^{\dagger}\right]=\left[R^{2}, A_{F}^{\dagger}\right]=2 N \ell_{B}^{2} .
$$

These relations imply that both $A_{B}^{\dagger}$ and $A_{F}^{\dagger}$ push each of the particles on the Laughlin state outwards by $\delta R=\sqrt{2} \ell_{B}$, to generate a quasihole (or a new magnetic cell of the area $2 \pi \ell_{B}^{2}$ ) at the origin. Hence, the bosonic and the fermionic quasiholes carry the identical fractional charge $1 / \mathrm{m}$. This may be regarded as a consequence of supersymmetry, because superpartners should have the same quantum numbers, such as mass and charge, except for spin. The commutation relations with the angular momentum are different,

$$
\left[L_{\perp}, A_{B}^{\dagger}\right]=N, \quad\left[L_{\perp}, A_{F}^{\dagger}\right]=\frac{N}{2},
$$


which implies that $A_{B}^{\dagger}$ does not change the spin of each particle, while $A_{F}^{\dagger}$ changes the spin from 0 to $-1 / 2$ [Fig. 3].

Similarly, bosonic and fermionic quasiparticle wave functions would be constructed by operating the annihilation operators

$$
A_{B}=\prod_{p} \frac{\partial}{\partial z_{p}}, \quad A_{F}=\prod_{p} \frac{\partial}{\partial \theta_{p}}
$$

on the Vandermonde determinant of $\Psi_{\text {Llin }} . A_{B}$ and $A_{F}$ satisfy the commutation relations with the radius operator

$$
\left[R^{2}, A_{B}\right]=\left[R^{2}, A_{F}\right]=-2 N \ell_{B}^{2},
$$

and with the angular momentum

$$
\left[L_{\perp}, A_{B}\right]=-N, \quad\left[L_{\perp}, A_{F}\right]=-\frac{N}{2} .
$$

Thus, $A_{B}$ attracts each of the particles on the Laughlin state by $\delta R=\sqrt{2} \ell_{B}$ inwards without changing the spin, and a bosonic quasiparticle with charge $-1 / m$ is generated at the origin. However, the operation of $A_{F}$ on the Vandermonde determinant of $\Psi_{\text {Llin }}$ yields zero, and fermionic quasiparticle excitations do not appear in the LLL. It is because, while $A_{F}$ changes the spin of each particle from 0 to $+1 / 2$, such spin- $1 / 2$ up particles are excluded due to the superchiral property in the LLL.

$A_{B}$ and $A_{B}^{\dagger}$ satisfy the bosonic commutation relations

$$
\begin{array}{r}
{\left[A_{B}, A_{B}^{\dagger}\right]=1,} \\
{\left[A_{B}, A_{B}\right]=\left[A_{B}^{\dagger}, A_{B}^{\dagger}\right]=0,}
\end{array}
$$

while $A_{F}$ and $A_{F}^{\dagger}$ satisfy "fermionic" commutation rela-

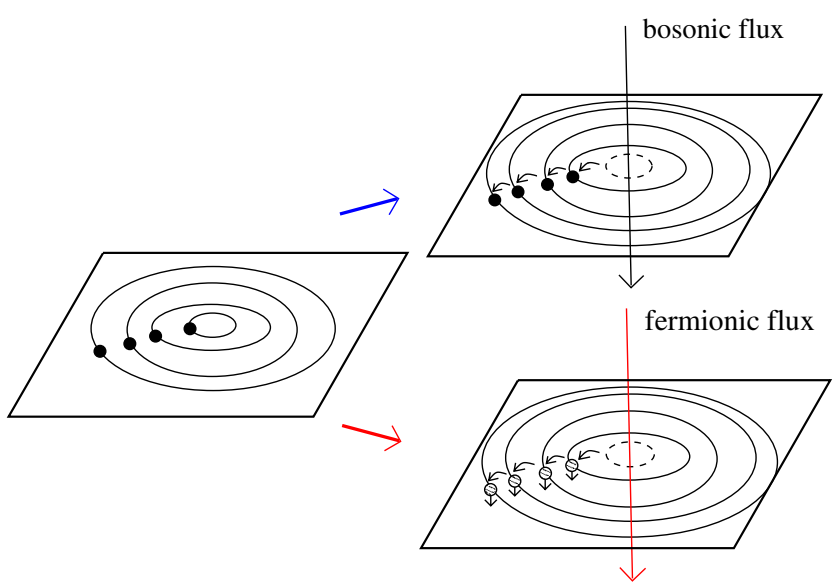

FIG. 3 (color online). In the left figure, the black blobs represent the spinless particles described by the Laughlin state for $\nu=1$. Because of the flux penetration, the spinless particles are pushed outwards by $\delta R=\sqrt{2} \ell_{B}$, and a quasihole is generated at the origin. The penetration of the bosonic flux keeps the particles spinless, while the penetration of the fermionic flux changes the spin of each particle from 0 to $-1 / 2$. tions

$$
\begin{array}{r}
A_{F} A_{F}^{\dagger}+(-1)^{N} A_{F}^{\dagger} A_{F}=1,(6.10 \mathrm{a}) \\
\left\{A_{F}, A_{f}\right\}=\left\{A_{F}^{\dagger}, A_{F}^{\dagger}\right\}=0 .(6.10 \mathrm{~b})
\end{array}
$$

\section{RELATIONS TO BILAYER QH SYSTEMS}

It is well known that fermionic harmonic oscillators can be regarded as the spin-1/2 ladder operators in supersymmetric quantum mechanics. In fact, the ladder operators made by Pauli matrices, $\left(\sigma_{+}, \sigma_{-}\right)=\frac{1}{2}\left(\sigma_{1}+i \sigma_{2}, \sigma_{1}-\right.$ $\left.i \sigma_{2}\right)$, satisfy the equations

$$
\left\{\sigma_{+}, \sigma_{-}\right\}=1, \sigma_{+}^{2}=\sigma_{-}^{2}=1,
$$

which are equivalent to the properties of the fermionic harmonic oscillators. Because of this identification, it is possible to map a supersymmetric harmonic oscillator system to a spin system. In the supersymmetric QH system, there exist two kinds of fermionic harmonic oscillators, $\left(\alpha, \alpha^{\dagger}\right)$ and $\left(\beta, \beta^{\dagger}\right)$. Therefore, in its corresponding spin system, two kinds of "spins" are needed. One possible candidate to meet this requirement is a bilayer $\mathrm{QH}$ system, where electrons carry not only their intrinsic spins but also pseudospins which specify double layers. By regarding the $\alpha$ "spin" as a pseudospin and the $\beta$ spin as an intrinsic spin, there exists a mapping to bilayer $\mathrm{QH}$ systems [Table I]. However, unfortunately, the real boson-fermion symmetry in the supersymmetric QH system is lost in this mapping, since the corresponding $\mathcal{N}=1$ nondynamical supersymmetry in the bilayer QH system acts as an interchange of the spin-1/2 up and down fermions.

When, we assign the $\alpha$ spin as

$$
\left(\alpha, \alpha^{\dagger}\right) \leftrightarrow\left(\tau_{+}, \tau_{-}\right) \equiv \frac{1}{2}\left(\tau_{2}+i \tau_{3}, \tau_{2}-i \tau_{3}\right),
$$

where $\left\{\tau_{a}\right\}(a=1,2,3)$ represent Pauli matrices for the pseudospin, the Hamiltonian (3.13) is rewritten as

$$
H=\omega\left(a^{\dagger} a+\frac{1}{2}\right)-\frac{\omega}{2} \tau_{1},
$$

which is the non-Coulomb part of the Hamiltonian for

TABLE I. The supersymmetric QH system is mapped to a bilayer $\mathrm{QH}$ system. The fermionic operators $\left(\alpha, \alpha^{\dagger}\right)$ and $\left(\beta, \beta^{\dagger}\right)$ are regarded as the ladder operators for pseudospin and intrinsic spin. The bosonic operators $\left(a, a^{\dagger}\right)$ and $\left(b, b^{\dagger}\right)$ are identified with the ladder operators for LLs and radially symmetric orbits.

\begin{tabular}{lc}
\hline \hline Supersymmetric QH system & Bilayer QH system \\
\hline Bosonic oscillator $a$ & Landau levels \\
Fermionic oscillator $\alpha$ & Bilayers or pseudospins \\
Bosonic oscillator $b$ & Radially symmetric orbits \\
Fermionic oscillator $\beta$ & (Intrinsic) spins \\
\hline \hline
\end{tabular}


bilayer QH systems, with tunneling interaction $\Delta_{\mathrm{SAS}}=\omega$ and without Zeeman interaction $\Delta_{Z}=0$. The LLL in supersymmetric $\mathrm{QH}$ systems can be regarded as the LLL of a symmetric layer state in bilayer $\mathrm{QH}$ systems. The Hamiltonian (7.3) appears in many different contexts of supersymmetric quantum mechanical systems, such as Pauli Hamiltonian with gyromagnetic factor 2 [42] and the Jaynes-Cummings model without interaction terms used in quantum optics [43]. However, it must be noted that each of such systems possesses $\mathcal{N}=1$ supersymmetry, while the present $\mathrm{QH}$ system has larger $\mathcal{N}=2$ supersymmetry due to the existence of extra $\mathcal{N}=1$ nondynamical supersymmetry.

\section{SUMMARY AND DISCUSSION}

Based on the supersymmetric NC algebra, we constructed QH liquids on a NC superplane. The supersymmetric Landau model enjoys (complex) $\mathcal{N}=2$ supersymmetry, one of which is dynamical and the other nondynamical. In the LLL, only the $\mathcal{N}=1$ nondynamical supersymmetry remains valid. Unlike ordinary supersymmetric quantum mechanics, the present supersymmetry represents a real boson-fermion symmetry. The NAC fermionic coordinates are related to spin degrees of freedom, and bring the superchiral property to the LLL. Since, on the NC superplane, the bosonic and the fermionic center-ofmass coordinates are decoupled, the Laughlin wave function and topological excitations have their superpartners unlike the QH liquid on the fuzzy supersphere. With use of the identification between the fermionic harmonic operators and the spin-1/2 ladder operators, supersymmetric $\mathrm{QH}$ systems are mapped to bilayer QH systems. In this mapping, the LLL in supersymmetric QH systems is regarded as the LLL in the symmetric layer state of bilayer QH systems.

While we have clarified bulk properties in the supersymmetric QH liquid, it is also important to study its edge excitations and effective field theory for further understanding of physics of the NAC geometry. We would like to pursue them in a future publication.

\section{ACKNOWLEDGMENTS}

I would like to acknowledge Satoshi Iso and Hiroshi Umetsu for useful discussions. I also thank Bernard deWitt, Shinsuke Kawai, and Giovanni Landi for helpful conversations.

\section{APPENDIX A: MAGNETIC TRANSLATIONS ON THE SUPERPLANE}

In this section, we summarize the Aharonov-Bohm phase accompanied by magnetic translation on the $\mathrm{NC}$ superplane. With use of the center-of-mass coordinates $\left(X_{i}, \Theta_{\alpha}\right)$, the supersymmetric magnetic translation operator is constructed as

$$
\mathcal{T}_{K}=e^{i\left(k_{i} X_{i}+\kappa_{\alpha} \Theta_{\alpha}\right)},
$$

which satisfies

$$
\mathcal{T}_{K} \cdot \mathcal{T}_{T}=\mathcal{T}_{K+T} e^{-(1 / 2) \ell_{B}^{2} \Sigma_{I J} K_{I} T_{J}},
$$

where $K \equiv\left(k_{i}, \kappa_{\alpha}\right), T \equiv\left(t_{i}, \tau_{\alpha}\right)$, and

$$
\Sigma \equiv\left(\begin{array}{cc}
\sigma_{2} & 0 \\
0 & -\sigma_{1}
\end{array}\right)
$$

The algebra for the supermagnetic translation is given by

$$
\begin{aligned}
{\left[\mathcal{T}_{K}, \mathcal{T}_{T}\right]=} & -2 \mathcal{T}_{K+T} \cdot \sinh \left(\frac{1}{2} \Sigma_{I J} K_{I} T_{J}\right) \\
= & 2 \mathcal{T}_{K+T} \cdot\left[e^{-(1 / 2) \ell_{B}^{2}\left(\sigma_{2}\right)_{i j} k_{i} t_{j}} \sinh \left(\frac{1}{2} \ell_{B}^{2}\left(\sigma_{1}\right)_{\alpha \beta} \kappa_{\alpha} \tau_{\beta}\right)\right. \\
& \left.+i e^{-(1 / 2) \ell_{B}^{2}\left(\sigma_{1}\right)_{\alpha \beta} \kappa_{\alpha} \tau_{\beta}} \sin \left(\frac{1}{2} \ell_{B}^{2} \epsilon_{i j} k_{i} t_{j}\right)\right] .
\end{aligned}
$$

The round-trip acquires a supersymmetric AharonovBohm phase as

$$
\mathcal{T}_{-K} \mathcal{T}_{-T} \mathcal{T}_{K} \mathcal{T}_{T}=e^{-\mathrm{BS}},
$$

where

$$
S \equiv \ell_{B}^{4} \Sigma_{I J} K_{I} T_{J}=\ell_{B}^{4}\left(\left(\sigma_{2}\right)_{i j} k_{i} p_{j}-\left(\sigma_{1}\right)_{\alpha \beta} \kappa_{\alpha} \tau_{\beta}\right),
$$

which represents the "area" on the superplane.

\section{APPENDIX B: INFINITE SYMMETRIES IN THE LLL}

It is well known that, in the LLL, infinite conserved charges appear and form the $W_{\infty}$ algebra $[4,44]$. Similarly, a supersymmetric extension of the $W_{\infty}$ algebra appears in the LLL of the supersymmetric QH systems. It is obvious that the following quantities commute with the Hamiltonian (3.13),

$$
\begin{aligned}
L_{m, n}^{B} & =\left(b^{\dagger}\right)^{m+1} b^{n+1}, \\
L_{m, n}^{F} & =\left(b^{\dagger}\right)^{m+1} b^{n+1} \beta, \\
L^{F \dagger}{ }_{m, n} & =\left(b^{\dagger}\right)^{n+1} b^{m+1} \beta^{\dagger},
\end{aligned}
$$

and $L^{B \dagger}{ }_{m, n}=L_{n, m}^{B}$, where $m, n \geq-1$. In particular, nondynamical supercharges are identified as $\left(\tilde{Q}, \tilde{Q}^{\dagger}\right)=$ $\left(L_{0,-1}^{F}, L^{F \dagger}{ }_{0,-1}\right) . L_{m, n}^{B}$ and $L_{k, l}^{F}$ satisfy a supersymmetric extension of the $W_{\infty}$ algebra as

$$
\begin{aligned}
& {\left[L_{m, n}^{B}, L_{k, l}^{B}\right]=\sum_{s=0}^{\operatorname{Min}(n, k)} \frac{(n+1) !(k+1) !}{(n-s) !(k-s) !(s+1) !}} \\
& \times L_{m+k-s, n+l-s}^{B}-((m, n) \leftrightarrow(k, l)), \\
& {\left[L_{m, n}^{B}, L_{k, l}^{F}\right]=\sum_{s=0}^{\operatorname{Min}(n, k)} \frac{(n+1) !(k+1) !}{(n-s) !(k-s) !(s+1) !}} \\
& \times L_{m+k-s, n+l-s}^{F}-((m, n) \leftrightarrow(k, l)), \\
& \left\{L_{m, n}^{F}, L_{k, l}^{F}\right\}=0 \text {. }
\end{aligned}
$$


$L_{m, n}^{B}$ and $L_{k, l}^{F}{ }^{\dagger}$ satisfy another supersymmetric $W_{\infty}$ algebra similar to Eq. (B2). The commutation relations with the angular momentum and the radius operator are given by

$$
\begin{aligned}
{\left[L_{\perp}, L_{m, n}^{B}\right] } & =(m-n) L_{m, n}^{B}, \\
{\left[L_{\perp}, L_{m, n}^{F}\right] } & =\left(m-n-\frac{1}{2}\right) L_{m, n}^{F}, \\
{\left[L_{\perp}, L^{F \dagger}{ }_{m, n}\right] } & =\left(-m+n+\frac{1}{2}\right) L_{m, n}^{F \dagger}{ }_{m},
\end{aligned}
$$

and

$$
\begin{aligned}
{\left[R^{2}, L_{m, n}^{B}\right] } & =(m-n) L_{m, n}^{B}, \\
{\left[R^{2}, L_{m, n}^{F}\right] } & =(m-n-1) L_{m, n}^{F}, \\
{\left[R^{2}, L^{F \dagger}{ }_{m, n}\right] } & =(-m+n+1) L_{m, n}^{F \dagger} .
\end{aligned}
$$

These relations imply that radially symmetric orbits (4.4) are related by $L_{m, n}^{B}, L_{m, n}^{F}$, and $L^{F \dagger}{ }_{m, n}$ as

$$
\begin{aligned}
|m+1 / 2\rangle & =\frac{1}{\sqrt{m ! n !}} L_{m-1, n-1}^{B}|n+1 / 2\rangle, \\
|m+1\rangle & =\frac{1}{\sqrt{(m+1) !(n+1) !}} L_{m, n}^{B}|n+1\rangle,
\end{aligned}
$$

and

$$
\begin{aligned}
|m+1 / 2\rangle & =\frac{1}{\sqrt{m !(n+1) !}} L_{n, m-1}^{F \dagger}|n+1\rangle, \\
|m+1\rangle & =\frac{1}{\sqrt{(m+1) ! n !}} L_{m, n-1}^{F}|n+1 / 2\rangle .
\end{aligned}
$$

[1] L. Susskind, hep-th/0101029.

[2] A. P. Polychronakos, J. High Energy Phys. 04 (2001) 011.

[3] S. M. Girvin and T. Jach, Phys. Rev. B 29, 5617 (1984).

[4] S. Iso, D. Karabali, and B. Sakita, Phys. Lett. B 296, 143 (1992).

[5] K. Moon, H. Mori, K. Yang, S. M. Girvin, A. H. MacDonald, L. Zheng, D. Yoshioka, and S.-C. Zhang, Phys. Rev. B 51, 5138 (1995).

[6] Z.F. Ezawa, G. Tsitsishvili, and K. Hasebe Phys. Rev. B 67, 125314 (2003).

[7] S. C. Zhang and J.P. Hu, Science 294, 823 (2001).

[8] J. Hu and S.C. Zhang, Phys. Rev. B 66, 125301 (2002).

[9] Y. Kimura, Nucl. Phys. B637, 177 (2002); B664, 512 (2003).

[10] B. A. Bernevig, C.-H. Chern, J.-P. Hu, N. Toumbas, and S.-C. Zhang, Ann. Phys. (N.Y.) 300, 185 (2002).

[11] Y.-X. Chen, hep-th/0209182; hep-th/0210059.

[12] George Sparling, cond-mat/0211679.

[13] Yi-Xin Chen, Bo-Yu Hou, and Bo-Yuan Hou, Nucl. Phys. B638, 220 (2002).

[14] H. Elvang and J. Polchinski, hep-th/0209104.

[15] Y.D. Chong and R. B. Laughlin, Ann. Phys. (N.Y.) 308, 237 (2003).

[16] S. Bellucci, P.-Y. Casteill, and A. Nersessian, Phys. Lett. B 574, 121 (2003).

[17] D. Karabali and V. P. Nair, Nucl. Phys. B641, 533 (2002).

[18] M. Fabinger, J. High Energy Phys. 05 (2002) 037.

[19] B. A. Bernevig, J.P. Hu, N. Toumbas, and S.C. Zhang, Phys. Rev. Lett. 91, 236803 (2003).

[20] G. Meng, J. Phys. A 36, 9415 (2003).

[21] K. Hasebe and Y. Kimura, Phys. Lett. B 602, 255 (2004).

[22] Y. Kimura, J. High Energy Phys. 04 (2004) 058.

[23] S. Murakami, N. Nagaosa, and S.-C. Zhang, Science 301, 1348 (2003); Phys. Rev. B 69, 235206 (2004).

[24] H. Ooguri and C. Vafa, Adv. Theor. Math. Phys. 7, 53 (2003); 7, 405 (2004).
[25] J. de Boer, P. A. Grassi, and P. van Nieuwenhuizen, Phys. Lett. B 574, 98 (2003).

[26] N. Seiberg, J. High Energy Phys. 06 (2003) 010.

[27] S. Iso and H. Umetsu, Phys. Rev. D 69, 105003 (2004).

[28] M. Hatsuda, S. Iso, and H. Umetsu, Nucl. Phys. B671, 217 (2003).

[29] E. Ivanov, L. Mezincescu, and P. K. Townsend, hep-th/ 0311159; hep-th/0404108.

[30] J. Gates, Jr., A. Jellal, E. H. Saidi, and M. Schreiber, J. High Energy Phys. 11 (2004) 075.

[31] K. Hasebe and Y. Kimura, Nucl. Phys. B709, 94 (2005).

[32] K. Hasebe, Phys. Rev. Lett. 94, 206802 (2005).

[33] R. Britto, B. Feng, and S-J. Rey, J. High Energy Phys. 08 (2003) 001.

[34] S. Ferrara, M. A. Lledo, and O. Macia, J. High Energy Phys. 09 (2003) 068.

[35] T. Araki, K. Ito, and A. Ohtsuka, Phys. Lett. B 573, 209 (2003).

[36] L. Lapointe, H. Ujino, and L. Vinet, Ann. Phys. (N.Y.) 314, 464 (2004).

[37] J. Lukierski, P.C. Stichel, and W. J. Zakrzewski, Phys. Lett. B 602, 249 (2004).

[38] Z.F. Ezawa, Quantum Hall Effects: Field Theoretical Approach and Related Topics (World Scientific, Singapore, 2000), Sect. 10.4.

[39] H. Nicolai, J. Phys. A 9, 1497 (1976).

[40] G. Junker, Supersymmetric Methods in Quantum and Statistical Physics (Springer, New York, 1996).

[41] R. B. Laughlin, Phys. Rev. Lett. 50, 1395 (1983).

[42] M. de Crombrugghe and V. Rittenberg, Ann. Phys. (N.Y.) 151, 99 (1983).

[43] V. A. Andreev and P. B. Lerner, Phys. Lett. A 134, 507 (1989); C. J. Lee, Phys. Lett. A 145, 177 (1990).

[44] A. Cappelli, C. A. Trugenberger, and G. R. Zemba, Nucl. Phys. B396, 465 (1993). 